\title{
A Domain Decomposition method based on iterative Operator Splitting method
}

\author{
Jürgen Geiser ${ }^{1}$ and Christos Kravvaritis ${ }^{2}$ \\ 1 Weierstrass Institute of Applied Analysis and Stochastics, \\ Mohrenstrasse 39, D-10117 Berlin, Germany \\ geiser@wias-berlin.de \\ 2 Department of Mathematics, University of Athens, \\ Panepistimiopolis 15784, Athens, Greece \\ ckrav@math.uoa.gr
}

\begin{abstract}
In this article a new approach is proposed for constructing domain decomposition methods based on iterative operator splitting methods. We study the convergence properties of such a method. The main feature of the method is the decoupling the space and time dimension. We confirm with two numerical applications the effectiveness of the proposed iterative operator splitting method in comparison with classical Schwarz waveform relaxation method as a standard method for domain decomposition. We provide improved results and convergence rates. The efficiency of considering the whole domain in the case of the iterative operator splitting method allows more accurate results.
\end{abstract}

Keywords. Operator Splitting method, Schwarz waveform relaxation method, domain decomposition methods, convergence analysis, multi-physics problems.

AMS subject classifications. 80A20, 80M25, 74S10, 76R50, 35J60, 35J65, 65M99, 65Z05.

\section{Introduction}

Iterative operator splitting methods are well-known splitting methods for complicated equations and do not allow a decoupling in separate equations, like the standard A-B splitting.

Overlapping Schwarz waveform relaxation is the name for a combination of two standard algorithms, Schwarz alternating method and wave form relaxation algorithm, which solves evolution problems in parallel. The method is characterized by the partitioning of the spatial domain into overlapping sub-domains, as in the classical Schwarz method.

The combined time-space iterative operator splitting method combines the Schwarz waveform relaxation and the iterative operator splitting method.

The outline of the paper is as follows. For our mathematical model we describe the convection-diffusion-reaction equation in section 2. The Fractional 
Splitting is introduced in section 3. We present the error analysis of the overlapping Schwarz waveform relaxation method for the solution of convectiondiffusion-reaction equation in section 4. Sections 5,6 and 7 provide all the necessary theoretical background and facts about the proposed new method. In section 8 we present the numerical results from the solution of selected model problems. We end the article in Section 9 with conclusion and comments.

\section{Mathematical Model and Methods}

\subsection{Model-Problem}

The motivation for the study presented below originates from a computational simulation of heat-transfer [5] and convection-diffusion-reaction-equations [4], $[7],[8]$ and $[6]$.

In our paper we concentrate on an one dimensional convection-diffusionreaction equation as our model problem given by

$$
\begin{aligned}
& u_{t}-D u_{x x}+\nu u_{x}=-\lambda u, \text { in } \Omega \times(0, T), \\
& u(x, 0)=u_{0}, \text { (Initial-Condition), } \\
& u(x, t)=u_{1}, \text { on } \partial \Omega \times(0, T), \text { (Dirichlet-Boundary-Condition) } .
\end{aligned}
$$

The unknown $u=u(x, t)$ is considered in $\Omega \times(0, T) \subset \mathbb{R} \times \mathbb{R}$, where $\Omega=[0, L]$. The constants $u_{0}, u_{1} \in \mathbb{R}^{+}$are used as initial and boundary parameters respectively. $\lambda$ is a constant factor, for example a decay-rate of a chemical reaction. $D$ is constant factor, for example the diffusion factor of a transport-process and $\nu$ is a constant factor, for example the velocity-rate of a transport-process.

The aim of this paper is to present a new method based on a mixed discretization method with Fractional-Splitting and Domain decomposition methods for the effective solution of strong coupled parabolic differential equations.

In the next section we discuss the decoupling of the time-scale with a first order fractional splitting-method.

\section{Fractional Splitting methods of first order for linear equations}

First we describe the simplest operator-splitting, which is called sequential operator splitting for the following linear system of ordinary differential equations:

$$
\partial_{t} u(t)=A u(t)+B u(t), \quad t \in(0, T),
$$

where the initial condition is $u(0)=u_{0}$. The operators $A$ and $B$ are spatially discretised operators, i.e. they correspond to the discretised in space convection and diffusion operators (matrices). Hence, they can be considered as bounded operators. 
The sequential operator-splitting method is introduced as a method that solves two sub-problems sequentially, where the different sub-problems are connected via the initial conditions. This means that we replace the original problem (1) with the sub-problems

$$
\begin{aligned}
& \frac{\partial u^{*}(t)}{\partial t}=A u^{*}(t), \quad \text { with } u^{*}\left(t^{n}\right)=u^{n}, \\
& \frac{\partial u^{* *}(t)}{\partial t}=B u^{* *}(t), \quad \text { with } u^{* *}\left(t^{n}\right)=u^{*}\left(t^{n+1}\right),
\end{aligned}
$$

where the splitting time-step is defined as $\tau_{n}=t^{n+1}-t^{n}$. The approximated split solution is defined as $u^{n+1}=u^{* *}\left(t^{n+1}\right)$.

Clearly, the replacement of the original problem with the sub-problems usually results some error, called splitting error. The splitting error of the sequential operator splitting method can be derived as follows (cf. e.g. [9], [10])

$$
\begin{aligned}
\rho_{n} & =\frac{1}{\tau_{n}}\left(\exp \left(\tau_{n}(A+B)\right)-\exp \left(\tau_{n} B\right) \exp \left(\tau_{n} A\right)\right) u\left(t^{n}\right) \\
& = \begin{cases}0, & \text { for }[A, B]=0 \\
O\left(\tau_{n}\right), & \text { for }[A, B] \neq 0,\end{cases}
\end{aligned}
$$

where $[A, B]:=A B-B A$ is the commutator of $A$ and $B$. Consequently, the splitting error is $O\left(\tau_{n}\right)$ when the operators $A$ and $B$ do not commute, otherwise the method is exact. Hence, by definition, the sequential operator splitting is called first order splitting method.

\section{Overlapping Schwarz wave form relaxation for the solution of convection-diffusion-reaction equation}

In this section we shall present the necessary conditions for the convergence of the overlapping Schwarz wave form relaxation method for the solution of the convection-diffusion-reaction equation with constant coefficients. We will utilize the convergence analysis for the solution of the decoupled and coupled system of convection reaction diffusion equation to elaborate the impact of the coupling on the convergence of the overlapping Schwarz wave form relaxation.

Given is the following model problem

$$
\begin{aligned}
& u_{t}+L u=f, \text { in } \Omega \times(0, T), \bar{\Omega} \times(0, T):=\bar{\Omega}_{1} \times(0, T) \cup \bar{\Omega}_{2} \times(0, T), \\
& u(x, 0)=u_{0},(\text { Initial-Condition }), \\
& u=g, \text { on } \partial \Omega \times(0, T),
\end{aligned}
$$

where $L$ denotes for each time $t$ a second-order partial differential operator $L u=-\nabla D \nabla u+v \nabla u+c u$ for the given coeffients $D \in \mathbb{R}^{+}, v \in \mathbb{R}^{n}, c \in \mathbb{R}^{+}$, and $n$ is the dimension of the space. The underlying domains $\Omega_{1}$ and $\Omega_{2}$ are 
nonconvex and lipschitzian and do not influence the following analysis. Each iteration step consists of two half steps associated with the two subdomains and we solve 2 subproblems

$$
\begin{aligned}
& u_{1 t}+L u_{1}^{n}=f, \text { in } \Omega_{1} \times(0, T), \\
& u_{1}(x, 0)=u_{10},(\text { Initial-Condition }), \\
& u_{1}^{n}=u_{2}^{n-1}, \text { on } L_{2}=\partial \Omega_{1} \times(0, T) \backslash \partial \Omega \times(0, T), \\
& u_{1}^{n}=g, \text { on } L_{0}=\partial \Omega \times(0, T) \cap \partial \Omega_{1} \times(0, T), \\
& u_{2 t}+L u_{2}^{n}=f, \text { in } \Omega_{2} \times(0, T), \\
& u_{2}(x, 0)=u_{20},(\text { Initial-Condition }), \\
& u_{2}^{n}=u_{1}^{n}, \text { on } L_{1}=\partial \Omega_{2} \times(0, T) \backslash \partial \Omega \times(0, T) ;, \\
& u_{2}^{n}=g, \text { on } L_{3}=\partial \Omega \times(0, T) \cap \partial \Omega_{2} \times(0, T),
\end{aligned}
$$

\subsection{Error of an Overlapping Schwarz wave form relaxation for the scalar convection-diffusion-reaction equation}

We consider the convection diffusion-reaction-equation, given by

$$
u_{t}=D u_{x x}-\nu u_{x}-\lambda u,
$$

defined on the domain $\Omega=[0, L]$ for $T=\left[T_{0}, T_{f}\right]$, with the following initial and boundary conditions

$$
u(0, t)=f_{1}(t), \quad u(L, t)=f_{2}(t), \quad u\left(x, T_{0}\right)=u_{0} .
$$

To solve the model problem using overlapping Schwarz wave form relaxation method, we divide the domain $\Omega$ in two overlapping sub-domains $\Omega_{1}=\left[0, L_{2}\right]$ and $\Omega_{2}=\left[L_{1}, L\right]$, where $L_{1}<L_{2}$ and $\Omega_{1} \bigcap \Omega_{2}=\left[L_{1}, L_{2}\right]$ is the overlapping region for $\Omega_{1}$ and $\Omega_{2}$.

Theorem 1. Let $\left\{e^{k+1}\right\}$ and $\left\{d^{k+1}\right\}$ be the sequences of errors from the solution of the subproblems (2) and (3) by Schwarz wave form relaxation over $\Omega_{1}$ and $\Omega_{2}$, respectively, then

$$
\left\|e^{k+2}\left(L_{1}, t\right)\right\|_{\infty} \leq \gamma\left\|e^{k}\left(L_{1}, t\right)\right\|_{\infty}
$$

and

$$
\left\|d^{k+2}\left(L_{2}, t\right)\right\|_{\infty} \leq \gamma\left\|d^{k}\left(L_{1}, t\right)\right\|_{\infty}
$$

where

$$
\gamma=\frac{\sinh \left(\beta L_{1}\right)}{\sinh \left(\beta L_{2}\right)} \frac{\sinh \left(\beta\left(L_{2}-L\right)\right)}{\sinh \left(\beta\left(L_{1}-L\right)\right)}<1,
$$

with $\beta=\frac{\sqrt{\nu^{2}+4 D \lambda}}{2 D}$.

Theorem 1 shows that the convergence of the overlapping Schwarz method depends on $\gamma=\frac{\sinh \left(\beta L_{1}\right)}{\sinh \left(\beta L_{2}\right)} \frac{\sinh \beta\left(L_{2}-L\right)}{\sinh \beta\left(L_{1}-L\right)}$. Due to the characteristic of the sinh function we will have sharp decay of the error for any $L_{1}<L_{2}$, and also for large size of overlapping the error will vanish. 


\section{The iterative splitting method}

The following algorithm is based on the iteration with fixed splitting discretization step-size $\tau$. On the time interval $\left[t^{n}, t^{n+1}\right]$ we solve the following subproblems consecutively for $i=0,2, \ldots 2 m$.

$$
\begin{aligned}
& \frac{\partial c_{i}(x, t)}{\partial t}=A c_{i}(x, t)+B c_{i-1}(x, t), \text { with } c_{i}\left(t^{n}\right)=c^{n} \\
& c_{0}\left(x, t^{n}\right)=c^{n}, c_{-1}=0, \\
& \text { and } c_{i}(x, t)=c_{i-1}(x, t)=c_{1}, \text { on } \partial \Omega \times(0, T), \\
& \frac{\partial c_{i+1}(x, t)}{\partial t}=A c_{i}(x, t)+B c_{i+1}(x, t), \\
& \text { with } c_{i+1}\left(x, t^{n}\right)=c^{n}, \\
& \text { and } c_{i}(x, t)=c_{i-1}(x, t)=c_{1}, \text { on } \partial \Omega \times(0, T),
\end{aligned}
$$

where $c^{n}$ is the known split approximation at the time level $t=t^{n}$, cf. [3].

\section{The combined time-space iterative splitting method}

Notation. For the sake of simplicity and for economy of space, from now on we omit writing the dependence of the functions on the variable $x$. However, it is important to leave the dependence on $t$ for obvious reasons.

The following algorithm iterates with fixed splitting discretization step-size $\tau$. On the time interval $\left[t^{n}, t^{n+1}\right]$ we solve the following sub-problems consecutively for $i=0,2, \ldots 2 m$ and $j=0,2, \ldots 2 q$. In this notation $i$ represents the iteration index for the time-splitting and $j$ represents the iteration index for the spatialsplitting.

$$
\begin{aligned}
& \frac{\partial c_{i, j}(t)}{\partial t}=\left.A\right|_{\Omega_{1}} c_{i, j}(t)+\left.A\right|_{\Omega_{2}} c_{i, j-1}(t)+\left.B\right|_{\Omega_{1}} c_{i-1, j}(t)+\left.B\right|_{\Omega_{2}} c_{i-1, j-1}(t), \\
& \text { with } c_{i, j}\left(t^{n}\right)=c^{n} \\
& \frac{\partial c_{i+1, j}(t)}{\partial t}=\left.A\right|_{\Omega_{1}} c_{i, j}(t)+\left.A\right|_{\Omega_{2}} c_{i, j-1}(t)+\left.B\right|_{\Omega_{1}} c_{i+1, j}(t)+\left.B\right|_{\Omega_{2}} c_{i-1, j-1}(t), \\
& \text { with } c_{i+1, j}\left(t^{n}\right)=c^{n} \\
& \frac{\partial c_{i, j+1}(t)}{\partial t}=\left.A\right|_{\Omega_{1}} c_{i, j}(t)+\left.A\right|_{\Omega_{2}} c_{i, j+1}(t)+\left.B\right|_{\Omega_{1}} c_{i+1, j}(t)+\left.B\right|_{\Omega_{2}} c_{i-1, j-1}(t), \\
& \text { with } c_{i, j+1}\left(t^{n}\right)=c^{n} \\
& \frac{\partial c_{i+1, j+1}(t)}{\partial t}=\left.A\right|_{\Omega_{1}} c_{i, j}(t)+\left.A\right|_{\Omega_{2}} c_{i, j+1}(t)+\left.B\right|_{\Omega_{1}} c_{i+1, j}(t)+\left.B\right|_{\Omega_{2}} c_{i+1, j+1}(t), \\
& \text { with } c_{i+1, j+1}\left(t^{n}\right)=c^{n}
\end{aligned}
$$

where $c^{n}$ is the known split approximation at the time level $t=t^{n}$, cf. [3]. 


\subsection{The nonoverlapping time-space iterative splitting method}

We introduce for the semi-discretisation in space the variable $k$ as the node for the point $x_{k}$ and we have $k \in(0, \ldots, p)$, where $p$ is the number of nodes. We have the decomposition in space, where $\Omega_{1}$ consists of the points $0, \ldots, p / 2$ and $\Omega_{2}$ of $p / 2+1, \ldots, p$, we assume $p$ is even. So we assume $\Omega_{1} \cap \Omega_{2}=\{\}$ and we have the following algorithm

$$
\begin{aligned}
& \frac{\partial\left(c_{i, j}\right)_{k}(t)}{\partial t}=\left.\tilde{A}\right|_{\Omega_{1}}\left(c_{i, j}\right)_{k}(t)+\left.\tilde{A}\right|_{\Omega_{2}}\left(c_{i, j-1}\right)_{k}(t) \\
& +\left.\tilde{B}\right|_{\Omega_{1}}\left(c_{i-1, j}\right)_{k}(t)+\left.\tilde{B}\right|_{\Omega_{2}}\left(c_{i-1, j-1}\right)_{k}(t), \\
& \text { with }\left(c_{i, j}\right)_{k}\left(t^{n}\right)=\left(c^{n}\right)_{k} \\
& \frac{\partial\left(c_{i+1, j}\right)_{k}(t)}{\partial t}=\left.\tilde{A}\right|_{\Omega_{1}}\left(c_{i, j}\right)_{k}(t)+\left.\tilde{A}\right|_{\Omega_{2}}\left(c_{i, j-1}\right)_{k}(t) \\
& +\left.\tilde{B}\right|_{\Omega_{1}}\left(c_{i+1, j}\right)_{k}(t)+\left.\tilde{B}\right|_{\Omega_{2}}\left(c_{i-1, j-1}\right)_{k}(t), \\
& \text { with }\left(c_{i+1, j}\right)_{k}\left(t^{n}\right)=\left(c^{n}\right)_{k} \\
& \frac{\partial\left(c_{i, j+1}\right)_{k}(t)}{\partial t}=\left.\tilde{A}\right|_{\Omega_{1}}\left(c_{i, j}\right)_{k}(t)+\left.\tilde{A}\right|_{\Omega_{2}}\left(c_{i, j+1}\right)_{k}(t) \\
& +\left.\tilde{B}\right|_{\Omega_{1}}\left(c_{i+1, j}\right)_{k}(t)+\left.\tilde{B}\right|_{\Omega_{2}}\left(c_{i-1, j-1}\right)_{k}(t), \\
& \text { with }\left(c_{i, j+1}\right)_{k}\left(t^{n}\right)=\left(c^{n}\right)_{k} \\
& \frac{\partial\left(c_{i+1, j+1}\right)_{k}(t)}{\partial t}=\left.\tilde{A}\right|_{\Omega_{1}}\left(c_{i, j}\right)_{k}(t)+\left.\tilde{A}\right|_{\Omega_{2}}\left(c_{i, j+1}\right)_{k}(t) \\
& +\left.\tilde{B}\right|_{\Omega_{1}}\left(c_{i+1, j}\right)_{k}(t)+\left.\tilde{B}\right|_{\Omega_{2}}\left(c_{i+1, j+1}\right)_{k}(t), \\
& \text { with }\left(c_{i+1, j+1}\right)_{k}\left(t^{n}\right)=\left(c^{n}\right)_{k}
\end{aligned}
$$

where $c^{n}$ is the known split approximation at the time level $t=t^{n}$, cf. [3].

The operators in the above equations are given as :

$$
\begin{gathered}
\left.\tilde{A}\right|_{\Omega_{1}}\left(c_{i, j}\right)_{k}=\left\{\begin{array}{c}
A c_{i, j} \quad \text { for } k \in\{0, \ldots, p / 2\} \\
0 \quad \text { for } k \in\{p / 2+1, \ldots, p\}
\end{array}\right. \\
\left.\tilde{A}\right|_{\Omega_{2}}\left(c_{i, j}\right)_{k}=\left\{\begin{array}{c}
0 \text { for } k \in\{0, \ldots, p / 2\} \\
A c_{i, j} \text { for } k \in\{p / 2, \ldots, p\}
\end{array}\right.
\end{gathered}
$$

Similar are the assignments for operator $B$.

$$
\begin{gathered}
\left.\tilde{B}\right|_{\Omega_{1}}\left(c_{i, j}\right)_{k}=\left\{\begin{array}{c}
B c_{i, j} \quad \text { for } k \in\{0, \ldots, p / 2\} \\
0 \quad \text { for } k \in\{p / 2+1, \ldots, p\}
\end{array}\right. \\
\left.\tilde{B}\right|_{\Omega_{2}}\left(c_{i, j}\right)_{k}=\left\{\begin{array}{cc}
0 & \text { for } k \in\{0, \ldots, p / 2\} \\
B c_{i, j} & \text { for } k \in\{p / 2, \ldots, p\}
\end{array}\right.
\end{gathered}
$$




\subsection{The overlapping time-space iterative splitting method}

We introduce for the semi-discretisation in space the variable $k$ as the node for the point $x_{k}$ and we have $k \in(0, \ldots, p)$, where $p$ is the number of nodes. Now we consider the overlapping case, so we assume $\Omega_{1} \cap \Omega_{2} \neq\{\}$. We have the following sets : $\Omega \backslash \Omega_{2}=\left\{0, \ldots, p_{1}\right\}, \Omega_{1} \cap \Omega_{2}=\left\{p_{1}+1, \ldots, p_{2}\right\}$ and $\Omega \backslash \Omega_{1}=\left\{p_{2}+1, \ldots, p\right\}$. We assume $p_{1}<p_{2}<p$ and can derive the following overlapping algorithm

$$
\begin{aligned}
& \frac{\partial\left(c_{i, j}\right)_{k}(t)}{\partial t}=\left.\tilde{A}\right|_{\Omega \backslash \Omega_{2}}\left(c_{i, j}\right)_{k}(t)+\left.\tilde{A}\right|_{\Omega_{1} \cap \Omega_{2}}\left(c_{i, j}, c_{i, j-1}\right)_{k}(t)+\left.\tilde{A}\right|_{\Omega \backslash \Omega_{1}}\left(c_{i, j-1}\right)_{k}(t) \\
& +\left.\tilde{B}\right|_{\Omega \backslash \Omega_{2}}\left(c_{i-1, j}\right)_{k}(t)+\left.\tilde{B}\right|_{\Omega_{1} \cap \Omega_{2}}\left(c_{i-1, j}, c_{i-1, j-1}\right)_{k}(t)+\left.\tilde{B}\right|_{\Omega \backslash \Omega_{1}}\left(c_{i-1, j-1}\right)_{k}(t), \\
& \text { with }\left(c_{i, j}\right)_{k}\left(t^{n}\right)=\left(c^{n}\right)_{k} \\
& \frac{\partial\left(c_{i+1, j}\right)_{k}(t)}{\partial t}=\left.\tilde{A}\right|_{\Omega \backslash \Omega_{2}}\left(c_{i, j}\right)_{k}(t)+\left.\tilde{A}\right|_{\Omega_{1} \cap \Omega_{2}}\left(c_{i, j}, c_{i, j-1}\right)_{k}(t)+\left.\tilde{A}\right|_{\Omega \backslash \Omega_{1}}\left(c_{i, j-1}\right)_{k}(t) \\
& +\left.\tilde{B}\right|_{\Omega \backslash \Omega_{2}}\left(c_{i+1, j}\right)_{k}(t)+\left.\tilde{B}\right|_{\Omega_{1} \cap \Omega_{2}}\left(c_{i+1, j}, c_{i-1, j-1}\right)_{k}(t)+\left.\tilde{B}\right|_{\Omega \backslash \Omega_{1}}\left(c_{i-1, j-1}\right)_{k}(t), \\
& \operatorname{with}\left(c_{i+1, j}\right)_{k}\left(t^{n}\right)=\left(c^{n}\right)_{k} \\
& \frac{\partial\left(c_{i, j+1}\right)_{k}(t)}{\partial t}=\left.\tilde{A}\right|_{\Omega \backslash \Omega_{2}}\left(c_{i, j}\right)_{k}(t)+\left.\tilde{A}\right|_{\Omega_{1} \cap \Omega_{2}}\left(c_{i, j+1}, c_{i, j}\right)_{k}(t)+\left.\tilde{A}\right|_{\Omega \backslash \Omega_{1}}\left(c_{i, j+1}\right)_{k}(t) \\
& +\left.\tilde{B}\right|_{\Omega \backslash \Omega_{2}}\left(c_{i+1, j}\right)_{k}(t)+\left.\tilde{B}\right|_{\Omega_{1} \cap \Omega_{2}}\left(c_{i+1, j}, c_{i-1, j-1}\right)_{k}(t)+\left.\tilde{B}\right|_{\Omega \backslash \Omega_{1}}\left(c_{i-1, j-1}\right)_{k}(t), \\
& \operatorname{with~}\left(c_{i, j+1}\right)_{k}\left(t^{n}\right)=\left(c^{n}\right)_{k} \\
& \frac{\partial\left(c_{i+1, j+1}\right)_{k}(t)}{\partial t}=\left.\tilde{A}\right|_{\Omega \backslash \Omega_{2}}\left(c_{i, j}\right)_{k}(t)+\left.\tilde{A}\right|_{\Omega_{1} \cap \Omega_{2}}\left(c_{i, j+1}, c_{i, j}\right)_{k}(t)+\left.\tilde{A}\right|_{\Omega \backslash \Omega_{1}}\left(c_{i, j+1}\right)_{k}(t) \\
& +\left.\tilde{B}\right|_{\Omega \backslash \Omega_{2}}\left(c_{i+1, j}\right)_{k}(t)+\left.\tilde{B}\right|_{\Omega_{1} \cap \Omega_{2}}\left(c_{i+1, j}, c_{i+1, j+1}\right)_{k}(t)+\left.\tilde{B}\right|_{\Omega \backslash \Omega_{1}}\left(c_{i+1, j+1}\right)_{k}(t), \\
& \text { with }\left(c_{i+1, j+1}\right)_{k}\left(t^{n}\right)=\left(c^{n}\right)_{k}
\end{aligned}
$$

where $c^{n}$ is the known split approximation at the time level $t=t^{n}$, cf. [3].

We have the operators :

$$
\begin{aligned}
& \left.\tilde{A}\right|_{\Omega \backslash \Omega_{2}}\left(c_{i, j}\right)_{k}=\left\{\begin{array}{cc}
A\left(c_{i, j}\right)_{k} & \text { for } k \in\left\{0, \ldots, p_{1}\right\} \\
0 & \text { for } k \in\left\{p_{1}+1, \ldots, p\right\}
\end{array}\right. \\
& \left.\tilde{A}\right|_{\Omega_{1} \cap \Omega_{2}}\left(c_{i, j}, c_{i, j+1}\right)_{k}=\left\{\begin{array}{cc}
A\left(\left(c_{i, j}+c_{i, j+1}\right) / 2\right)_{k} & \text { for } k \in\left\{p_{1}+1, \ldots, p_{2}\right\} \\
0 & \text { for } k \in\left\{p_{2}+1, \ldots, p\right\}
\end{array}\right. \\
& \left.\tilde{A}\right|_{\Omega \backslash \Omega_{1}}\left(c_{i, j}\right)_{k}=\left\{\begin{array}{cc}
0 & \text { for } k \in\left\{0, \ldots, p_{2}\right\} \\
A\left(c_{i, j}\right)_{k} & \text { for } k \in\left\{p_{2}+1, \ldots, p\right\}
\end{array}\right.
\end{aligned}
$$

Similar are the assignments for operator $B$.

$$
\begin{gathered}
\left.\tilde{B}\right|_{\Omega \backslash \Omega_{2}}\left(c_{i, j}\right)_{k}=\left\{\begin{array}{cc}
B\left(c_{i, j}\right)_{k} & \text { for } k \in\left\{0, \ldots, p_{1}\right\} \\
0 & \text { for } k \in\left\{p_{1}+1, \ldots, p\right\}
\end{array}\right. \\
\left.\tilde{B}\right|_{\Omega_{1} \cap \Omega_{2}}\left(c_{i, j}, c_{i, j+1}\right)_{k}=\left\{\begin{array}{cc}
B\left(\left(c_{i, j}+c_{i, j+1}\right) / 2\right)_{k} & \text { for } k \in\left\{p_{1}+1, \ldots, p_{2}\right\} \\
0 & \text { for } k \in\left\{p_{2}+1, \ldots, p\right\}
\end{array}\right.
\end{gathered}
$$




$$
\left.\tilde{B}\right|_{\Omega \backslash \Omega_{1}}\left(c_{i, j}\right)_{k}=\left\{\begin{array}{cc}
0 & \text { for } k \in\left\{0, \ldots, p_{2}\right\} \\
B\left(c_{i, j}\right)_{k} & \text { for } k \in\left\{p_{2}+1, \ldots, p\right\}
\end{array}\right.
$$

Dicretisation of the operators

The discretization of the operators is given as :

$$
\begin{gathered}
A\left(c_{i, j}\right)_{k}=D /(\Delta x)^{2}\left(-\left(c_{i, j}\right)_{k+1}+2\left(c_{i, j}\right)_{k}-\left(c_{i, j}\right)_{k-1}\right) \\
-v / \Delta x\left(\left(c_{i, j}\right)_{k}-\left(c_{i, j}\right)_{k-1}\right) \\
B\left(c_{i, j}\right)_{k}=\lambda\left(c_{i, j}\right)_{k} .
\end{gathered}
$$

\section{Error analysis and convergence of the combined method}

Theorem 2. Let us consider the nonlinear operator-equation in a Banach space $\mathrm{X}$

$$
\begin{gathered}
\partial_{t} c(t)=A_{1} c(t)+A_{2} c(t)+B_{1} c(t)+B_{2} c(t), \quad 0<t \leq T, \\
c(0)=c_{0},
\end{gathered}
$$

where $A_{1}, A_{2}, B_{1}, B_{2}, A_{1}+A_{2}+B_{1}+B_{2}: \mathbf{X} \rightarrow \mathbf{X}$ are given linear operators being generators of the $C_{0}$-semigroup and $c_{0} \in \mathbf{X}$ is a given element. Then the iteration process (6)-(9) is convergent and the rate of the convergence is one. We obtain the iterative result : $\left\|e_{i, j}(t)\right\| \leq K \tau_{n}\left\|e_{i-1, j-1}(t)\right\|$, where $\tau_{n}=t^{n+1}-$ $t^{n}$.

Proof. Let us consider the iteration (6)-(9) on the sub-interval $\left[t^{n}, t^{n+1}\right]$. We consider the case of the exact initial-conditions given as $c_{i, j}\left(t^{n}\right)=c_{0}$, a generalization is also possible. So for the error function $e_{i, j}(t):=c(t)-c_{i, j}(t)$ we have the relations

$$
\begin{aligned}
& \partial_{t} e_{i, j}(t)=A_{1} e_{i, j}(t)+A_{2} e_{i, j-1}(t)+B_{1} e_{i-1, j}(t)+B_{2} e_{i-1, j-1}(t), \\
& e_{i, j}\left(t^{n}\right)=0, \\
& \partial_{t} e_{i+1, j}(t)=A_{1} e_{i, j}(t)+A_{2} e_{i, j-1}(t)+B_{1} e_{i+1, j}(t)+B_{2} e_{i-1, j-1}(t), \\
& e_{i+1, j}\left(t^{n}\right)=0, \\
& \partial_{t} e_{i, j+1}(t)=A_{1} e_{i, j}(t)+A_{2} e_{i, j+1}(t)+B_{1} e_{i+1, j}(t)+B_{2} e_{i-1, j-1}(t), \\
& e_{i, j+1}\left(t^{n}\right)=0
\end{aligned}
$$

and

$$
\begin{aligned}
& \partial_{t} e_{i, j}(t)=A_{1} e_{i, j}(t)+A_{2} e_{i, j+1}(t)+B_{1} e_{i+1, j}(t)+B_{2} e_{i+1, j+1}(t), \\
& e_{i, j}\left(t^{n}\right)=0
\end{aligned}
$$


for $t \in\left[t^{n}, t^{n+1}\right], i, j=0,2,4, \ldots$, with $e_{0,0}(0)=0$ and $e_{-1,0}(t)=e_{0,-1}(t)=$ $e_{-1,-1}(t)=c(t)$.

In the following we use the notations $\mathbf{X}^{4}$ for the product space $\times_{i=1}^{4} \mathbf{X}$ enabled with the norm $\left\|\left(u_{1}, u_{2}, u_{3}, u_{4}\right)^{t}\right\|=\max _{i=1, \ldots, 4}\left\{\left\|u_{i}\right\|\right\}\left(u_{i} \in \mathbf{X}, i=1, \ldots, 4\right)$. The elements $\mathcal{E}_{i}(t), \mathcal{F}_{i}(t) \in \mathbf{X}^{4}$ and the linear operator $\mathcal{A}: \mathbf{X}^{4} \rightarrow \mathbf{X}^{4}$ are defined as follows

$$
\begin{gathered}
\mathcal{E}_{i, j}(t)=\left[\begin{array}{c}
e_{i, j}(t) \\
e_{i+1, j}(t) \\
e_{i, j+1}(t) \\
e_{i+1, j+1}(t)
\end{array}\right], \quad \mathcal{A}=\left[\begin{array}{llll}
A_{1} & 0 & 0 & 0 \\
A_{1} & B_{1} & 0 & 0 \\
A_{1} & B_{1} & A_{2} & 0 \\
A_{1} & B_{1} & A_{2} & B_{2}
\end{array}\right] \\
\mathcal{F}_{i, j}(t)=\left[\begin{array}{r}
A_{2} e_{i, j-1}(t)+B_{1} e_{i-1, j}(t)+B_{2} e_{i-1, j-1}(t) \\
A_{2} e_{i, j-1}(t)+B_{2} e_{i-1, j-1}(t) \\
B_{2} e_{i-1, j-1}(t) \\
0
\end{array}\right] .
\end{gathered}
$$

Using the notations (30), the relations (26)-(29) can be written in the form

$$
\begin{gathered}
\partial_{t} \mathcal{E}_{i, j}(t)=\mathcal{A E}_{i, j}(t)+\mathcal{F}_{i, j}(t), \quad t \in\left[t^{n}, t^{n+1}\right], \\
\mathcal{E}_{i, j}\left(t^{n}\right)=0 .
\end{gathered}
$$

We estimate the right hand side $\mathcal{F}_{i, j}(t)$ in the following Lemma :

Lemma 1. For $\mathcal{F}_{i, j}(t)$ it holds

$$
\left\|\mathcal{F}_{i, j}(t)\right\| \leq C\left\|e_{i-1, j-1}(t)\right\| .
$$

Proof. We have the following norm

$\left\|\mathcal{F}_{i, j}(t)\right\|=\max \left\{\left\|\mathcal{F}_{i, j, 1}(t)\right\|,\left\|\mathcal{F}_{i, j, 2}(t)\right\|,\left\|\mathcal{F}_{i, j, 3}(t)\right\|,\left\|\mathcal{F}_{i, j, 4}(t)\right\|\right\}$.

Each term can be estimated as:

$$
\begin{aligned}
\left\|\mathcal{F}_{i, j, 1}(t)\right\| & =\left\|A_{2} e_{i, j-1}(t)+B_{1} e_{i-1, j}(t)+B_{2} e_{i-1, j-1}(t)\right\| \leq C_{1}\left\|e_{i-1, j-1}(t)\right\| \\
\left\|\mathcal{F}_{i, j, 2}(t)\right\| & =\left\|A_{2} e_{i, j-1}(t)+B_{2} e_{i-1, j-1}(t)\right\| \leq C_{2}\left\|e_{i-1, j-1}(t)\right\| \\
\left\|\mathcal{F}_{i, j, 3}(t)\right\| & =\left\|B_{2} e_{i-1, j-1}(t)\right\| \leq C_{3}\left\|e_{i-1, j-1}(t)\right\|
\end{aligned}
$$

Based on the theorem of Fubini, see [1], for decoupable operators, we obtain: $\left\|e_{\tilde{i}, \tilde{j}}(t)\right\| \leq\left\|e_{i-1, j-1}\right\|$, for $\tilde{i}=\{i-1, i\}$ and $\tilde{j}=\{j-1, j\}$.

Hence,

$$
\left\|\mathcal{F}_{i, j}(t)\right\| \leq C\left\|e_{i-1, j-1}(t)\right\|,
$$

where $C$ is the maximum value of $C_{1}, C_{2}$ and $C_{3}$.

Using the variations of constants formula, the solution of the abstract Cauchy problem (31) with homogeneous initial condition can be written as

$$
\mathcal{E}_{i, j}(t)=\int_{t^{n}}^{t} \exp (\mathcal{A}(t-s)) \mathcal{F}_{i, j}(s) d s, \quad t \in\left[t^{n}, t^{n+1}\right] .
$$


(See, e.g. [2].) Hence, using the denotation

$$
\left\|\mathcal{F}_{i, j}\right\|_{\infty}=\sup _{t \in\left[t^{n}, t^{n+1}\right]}\left\|\mathcal{F}_{i, j}(t)\right\|,
$$

and taking into account Lemma 1, we have

$$
\begin{gathered}
\left\|\mathcal{E}_{i, j}(t)\right\| \leq\left\|\mathcal{F}_{i, j}\right\|_{\infty} \int_{t^{n}}^{t}\|\exp (\mathcal{A}(t-s))\| d s \\
\leq C\left\|e_{i-1, j-1}(t)\right\| \int_{t^{n}}^{t}\|\exp (\mathcal{A}(t-s))\| d s, \quad t \in\left[t^{n}, t^{n+1}\right] .
\end{gathered}
$$

Due to our linearity assumptions for the operators, $\mathcal{A}$ is a generator of the one-parameter $C_{0}$ semigroup $(\mathcal{A}(t))_{t \geq 0}$. Since $(\mathcal{A}(t))_{t \geq 0}$ is a semigroup therefore the so called growth estimation

$$
\|\exp (\mathcal{A} t)\| \leq \widetilde{K} \exp (\omega t) ; \quad t \geq 0,
$$

holds with some numbers $\widetilde{K} \geq 0$ and $\omega \in \mathbb{R}$, see [2].

- Assume that $(\mathcal{A}(t))_{t \geq 0}$ is a bounded or exponentially stable semigroup, i.e. (33) holds with some $\omega \leq 0$. Then obviously the estimate

$$
\|\exp (\mathcal{A} t)\| \leq \widetilde{K} ; \quad t \geq 0,
$$

holds, and considering (32), we have the relation

$$
\left\|\mathcal{E}_{i, j}(t)\right\| \leq K \tau_{n}\left\|e_{i-1, j-1}(t)\right\|, \quad t \in\left[t^{n}, t^{n+1}\right] .
$$

- Assume that $(\mathcal{A}(t))_{t \geq 0}$ has an exponential growth with some $\omega>0$. Intergrating (33) yields

$$
\int_{t^{n}}^{t}\|\exp (\mathcal{A}(t-s))\| d s \leq K_{\omega}(t), \quad t \in\left[t^{n}, t^{n+1}\right],
$$

where

$$
K_{\omega}(t)=\frac{\widetilde{K}}{\omega}\left(\exp \left(\omega\left(t-t^{n}\right)\right)-1\right), \quad t \in\left[t^{n}, t^{n+1}\right],
$$

and hence

$$
K_{\omega}(t) \leq \frac{\widetilde{K}}{\omega}\left(\exp \left(\omega \tau_{n}\right)-1\right)=\widetilde{K} \tau_{n}+\mathcal{O}\left(\tau_{n}^{2}\right),
$$

where $\tau_{n}=t^{n+1}-t^{n}$.

The estimations (34), (35) and (36) result in

$$
\left\|\mathcal{E}_{i, j}(t)\right\| \leq K \tau_{n}\left\|e_{i-1, j-1}(t)\right\|,
$$

where $K=\widetilde{K} \cdot C$ for both cases.

Taking into account the definition of $\mathcal{E}_{i, j}(t)$ and the norm $\|\cdot\|$, we obtain

$$
\left\|e_{i, j}(t)\right\| \leq K \tau_{n}\left\|e_{i-1, j-1}(t)\right\|,
$$

which proves our statement. 
Remark 1. We can generalise our results for $n$ decomposed domains. We obtain the same results for the generalised semi-group $\mathcal{A}: \mathbf{X}^{n} \rightarrow \mathbf{X}^{n}$.

Remark 2. We require the double amount of iterations due to the 2 partitions (i.e. $i$ for time, $j$ for space). Also, for higher order accuracy more amount of work is needed, e.g. $2(2 m+1)$ iterations for $O\left(\tau^{2 m+1}\right)$ accuracy.

\section{Numerical Results}

In this section we will present the numerical results from the solution of the convection-diffusion-reaction equation using several variations of the proposed methods in comparison with already known classical methods.

\subsection{First numerical example}

We consider the one-dimensional convection-reaction-diffusion equation

$$
\begin{aligned}
& \partial_{t} u+v \partial_{x} u-\partial_{x} D \partial_{x} u=-\lambda u, \text { in } \Omega \times\left(T_{0}, T_{f}\right), \\
& u(x, 0)=u_{e x}(x, 0),(\text { Initial-Condition }), \\
& u(x, t)=u_{e x}(x, t), \text { on } \partial \Omega \times\left(T_{0}, T_{f}\right),
\end{aligned}
$$

where $\Omega \times\left[T_{0}, T_{f}\right]=[0,150] \times\left[100,10^{5}\right]$.

The exact solution is given as

$$
u_{e x}(x, t)=\frac{u_{0}}{2 \sqrt{D \pi t}} \exp \left(-\frac{(x-v t)^{2}}{4 D t}\right) \exp (-\lambda t) .
$$

The initial condition and the Dirichlet boundary conditions are defined using the exact solution (40) at starting time $T_{0}=100$ and with $u_{0}=1.0$. We have $\lambda=10^{-5}, v=0.001$ and $D=0.0001$.

\subsection{Solution using classical methods}

A-B splitting combined with Schwarz wave form relaxation method In order to solve the model problem using overlapping Schwarz wave form relaxation method, we divide the domain $\Omega$ in two overlapping sub-domains $\Omega_{1}=\left[0, L_{2}\right]$ and $\Omega_{2}=\left[L_{1}, L\right]$, where $L_{1}<L_{2}$, and $\Omega_{1} \bigcap \Omega_{2}=\left[L_{1}, L_{2}\right]$ is the overlapping region for $\Omega_{1}$ and $\Omega_{2}$.

To start the wave form relaxation algorithm we consider first the solution of the model problem (47) over $\Omega_{1}$ and $\Omega_{2}$ as follows

$$
\begin{aligned}
& v_{t} \quad=D v_{x x}-\nu v_{x}-\lambda v \text { over } \Omega_{1}, \quad t \in\left[T_{0}, T_{f}\right] \\
& v(0, t)=f_{1}(t), \quad t \in\left[T_{0}, T_{f}\right] \\
& v\left(L_{2}, t\right)=w\left(L_{2}, t\right), \quad t \in\left[T_{0}, T_{f}\right] \\
& v\left(x, T_{0}\right)=u_{0} \quad x \in \Omega_{1}
\end{aligned}
$$




$$
\begin{aligned}
& w_{t}=D w_{x x}-\nu w_{x}-\lambda w \text { over } \Omega_{2}, \quad t \in\left[T_{0}, T_{f}\right] \\
& w\left(L_{1}, t\right)=v\left(L_{1}, t\right), \quad t \in\left[T_{0}, T_{f}\right] \\
& w(L, t)=f_{2}(t), \quad t \in\left[T_{0}, T_{f}\right] \\
& w\left(x, T_{0}\right)=u_{0} \quad x \in \Omega_{2}
\end{aligned}
$$

where $v(x, t)=\left.u(x, t)\right|_{\Omega_{1}}$ and $w(x, t)=\left.u(x, t)\right|_{\Omega_{2}}$.

Then the Schwarz wave form relaxation is given by

$$
\begin{aligned}
v_{t}^{k+1} & =D v_{x x}^{k+1}-\nu v_{x}^{k+1}-\lambda v^{k+1} \text { over } \Omega_{1}, \quad t \in\left[T_{0}, T_{f}\right] \\
v^{k+1}(0, t) & =f_{1}(t), \quad t \in\left[T_{0}, T_{f}\right] \\
v^{k+1}\left(L_{2}, t\right) & =w^{k}\left(L_{2}, t\right), \quad t \in\left[T_{0}, T_{f}\right] \\
v^{k+1}\left(x, T_{0}\right) & =u_{0} \quad x \in \Omega_{1}, \\
& =D w_{x x}^{k+1}-\nu w_{x}^{k+1}-\lambda w^{k+1} \text { over } \Omega_{2}, \quad t \in\left[T_{0}, T_{f}\right] \\
w_{t}^{k+1} \quad & \\
w^{k+1}\left(L_{1}, t\right) & =v^{k}\left(L_{1}, t\right), \quad t \in\left[T_{0}, T_{f}\right] \\
w^{k+1}(L, t) & =f_{2}(t), \quad t \in\left[T_{0}, T_{f}\right] \\
w^{k+1}\left(x, T_{0}\right) & =u_{0} \quad x \in \Omega_{2} .
\end{aligned}
$$

For the solution of (41) and (42) we will apply the sequential operator splitting method (A-B splitting). For this purpose we divide each of these two equations in terms of the operators $A=D \frac{\partial^{2}}{\partial x^{2}}-\nu \frac{\partial}{\partial x}$ and $B=-\lambda$. The splitting scheme for each of them is given in the following form:

$$
\begin{aligned}
& \frac{\partial u^{*}(x, t)}{\partial t}=D u_{x x}^{*}-\nu u_{x}^{*}, \quad \text { with } u^{*}\left(x, t^{n}\right)=u_{0} \\
& \frac{\partial u^{* *}(x, t)}{\partial t}=-\lambda u^{* *}(t), \quad \text { with } u^{* *}\left(x, t^{n}\right)=u^{*}\left(x, t^{n+1}\right),
\end{aligned}
$$

where $u^{*}(x, t)=u^{* *}(x, t)=u_{1}$, on $\partial \Omega \times(0, T)$, are the Dirichlet-BoundaryConditions for the equations. The solution is given as $u\left(x, t^{n+1}\right)=u^{* *}\left(x, t^{n+1}\right)$. We obtain an exact method because of commuting operators.

For the discretization of equation (43) we apply the finite-difference method for the spatial discretization and the implicite Euler method for the time discretization. The discretization is given as

$$
\begin{aligned}
& \frac{1}{t^{n+1}-t^{n}}\left(u^{*}\left(x_{i}, t^{n+1}\right)-u^{*}\left(x_{i}, t^{n}\right)\right) \\
& =D \frac{1}{h_{i}^{2}}\left(-u^{*}\left(x_{i+1}, t^{n+1}\right)+2 u^{*}\left(x_{i}, t^{n+1}\right)-u^{*}\left(x_{i-1}, t^{n+1}\right)\right) \\
& -\nu \frac{1}{h_{i}}\left(u^{*}\left(x_{i}, t^{n+1}\right)-u^{*}\left(x_{i-1}, t^{n+1}\right)\right), \\
& \quad \text { with } u^{*}\left(x_{1}, t^{n}\right)=u^{*}\left(x_{2}, t^{n}\right)=u_{0} \text { and } u^{*}\left(x_{0}, t^{n}\right)=u^{*}\left(x_{m}, t^{n}\right)=0 \\
& u^{* *}(x, t)=\exp \left(-\lambda\left(t-t^{n}\right) u^{*}\left(x, t^{n+1}\right),\right.
\end{aligned}
$$

where $h_{i}=x_{i+1}-x_{i}$ and we assume a partition with $m$-nodes.

We are interested in specifying the error between the solution obtained with the above described algorithm and the exact solution. We provide a variety of 
results for several sizes of space- and time-partition, and also for various overlap sizes. Precisely, we treat the cases $h=1,0.5,0.25$ as spatial step-size, $\Delta t=$ $5,10,20$ as time step. The considered subdomains are $\Omega_{1}=[0,60]$ and $\Omega_{2}=$ $[30,150]$ and $\Omega_{1}=[0,100]$ and $\Omega_{2}=[30,150]$, with overlap sizes 30 and 70 , respectively. Both the approximated and the exact solution are evaluated at the end-time $t=10^{5}$. The errors given in Table 1 are the maximum errors that occurred over the whole space domain, i.e. they are calculated using the $\infty$-norm for vectors.

\begin{tabular}{||c||c|c||c|c||c|c||}
\hline \hline time-step & err & err & err & err & err & err \\
\hline$\Delta t=5$ & $2.24 e-3$ & $1.28 e-3$ & $2.21 e-4$ & $2.20 e-4$ & $1.99 e-5$ & $1.97 e-5$ \\
\hline$\Delta t=10$ & $2.61 e-3$ & $2.56 e-3$ & $3.02 e-4$ & $3.01 e-4$ & $4.34 e-5$ & $4.29 e-5$ \\
\hline$\Delta t=20$ & $2.81 e-3$ & $2.73 e-3$ & $5.22 e-4$ & $5.14 e-4$ & $5.66 e-4$ & $4.88 e-4$ \\
\hline \hline overlap & 30 & 70 & 30 & 70 & 30 & 70 \\
\hline space-step & \multicolumn{2}{|c||}{$h=1$} & \multicolumn{2}{|c||}{$h=0.5$} & \multicolumn{2}{|c||}{$h=0.25$} \\
\hline
\end{tabular}

Table 1. Error for the scalar convection diffusion reaction-equation using the classical method for two different sizes of overlapping 30 and 70 .

\subsection{Solution using the proposed method}

For the solution of (37)-(39) with the combined time-space iterative splitting method we divide again the equation in terms of the operators $A=D \frac{\partial^{2}}{\partial x^{2}}-\nu \frac{\partial}{\partial x}$ and $B=-\lambda$. We will utilize the proposed scheme (14)-(25).

The index $k=0,1, \ldots p$ is associated with the subdomains, i.e. for $k=$ $0, \ldots, p / 2$ we are working on $\Omega_{1}$ and for $k=p / 2+1, \ldots, p$ on $\Omega_{2}$. For the first set of values for $k$ we have actually only the effect of the restrictions of the operators $A$ and $B$ on $\Omega_{1}$. Similarly, the second set of values for $k$ indicates the action of the restrictions of both operators on $\Omega_{2}$. The outline of the method in Section 6, which is also adopted here, is given without loss of generality for a subdomain-determining value $k=p / 2$, just for an overview. This crucial value is determined appropriately according to the three cases of the overlapping subdomains, which we examine in our experiments.

The indices $i$ and $j$ are related to the time- and space-discretization, respectively. For every $k=0, \ldots, p / 2$ and for every interval of the space-discretization we solve the appropriate problems on $\Omega_{1}$, for every interval of the time-discretization. Similarly for $k=p / 2+1, \ldots, p$ on $\Omega_{2}$.

From a software development point of view, the above described numerical scheme can be realized with three "for" loops. The first, outer loop is for all values of $k$. After this loop there exists a control for $k$, which discriminates two cases for $k<p / 2$ and for $k \geq p / 2+1$, and sets up the data of the algorithm appropriately for $\Omega_{1}$ or $\Omega_{2}$, respectively. The second, middle loop is running for all values of $i$ and the third, inner loop is for all values of $j$. 
By a closer examination of the scheme (14)-(17), taking into account the definitions (18)-(23), we observe that the problems to be solved in the innermost loop are of the form $\partial_{t} c=A c+B c, c\left(x, t^{n}\right)=c^{n}$, where $c$ appears with appropriate indices $i$ and $j$. These problems are solved with suitable modification and implementation of the iterative operator splitting scheme (4)-(5). The notion of the iterative process takes place in both time- and space-dimensions.

We are interested again in specifying the error between the solution obtained with the above described algorithm and the exact solution. We provide the same variety of results as in the previous subsection, so that a comparison between the proposed and classical methods can be established. Both the approximated and the exact solution are evaluated at the end-time $t=10^{5}$. The errors given in the following tables are the maximum errors that occurred over the whole space domain, i.e. they are calculated using the $\infty$-norm for vectors. The results are given in Table 2 .

\begin{tabular}{||c||c|c||c|c||c|c||}
\hline \hline time-step & err & err & err & err & err & err \\
\hline$\Delta t=5$ & $1.47 e-2$ & $3.49 e-3$ & $2.13 e-4$ & $1.54 e-4$ & $6.49 e-6$ & $8.29 e-6$ \\
\hline$\Delta t=10$ & $2.26 e-2$ & $7.46 e-3$ & $2.22 e-4$ & $2.15 e-4$ & $3.47 e-5$ & $3.37 e-5$ \\
\hline$\Delta t=20$ & $4.39 e-2$ & $1.20 e-2$ & $5.21 e-4$ & $4.53 e-4$ & $5.42 e-4$ & $3.21 e-4$ \\
\hline \hline overlap & 30 & 70 & 30 & 70 & 30 & 70 \\
\hline space-step & \multicolumn{2}{|c||}{$h=1$} & \multicolumn{2}{|c||}{$h=0.5$} & \multicolumn{2}{c||}{$h=0.25$} \\
\hline
\end{tabular}

Table 2 . Error for the scalar convection diffusion reaction-equation using the proposed method for two different sizes of overlapping 30 and 70 .

\subsection{Second numerical example}

We consider the two-dimensional convection-reaction-diffusion equation

$$
\begin{aligned}
& \partial_{t} u+v \partial_{x} u-\partial_{x} D_{x} \partial_{x} u-\partial_{y} D_{y} \partial_{y} u=-\lambda u, \text { in } \Omega \times\left(T_{0}, T_{f}\right), \\
& u(x, y, 0)=u_{e x}(x, y, 0),(\text { Initial-Condition }), \\
& u(x, y, t)=u_{e x}(x, y, t), \text { on } \partial \Omega \times\left(T_{0}, T_{f}\right),
\end{aligned}
$$

where $\Omega \times\left[T_{0}, T_{f}\right]=[0,150] \times[0,150] \times\left[100,10^{5}\right]$.

The exact solution is given as

$$
u_{e x}(x, y, t)=\frac{u_{0}}{4 \sqrt{D_{x} \pi t} \sqrt{D_{y} \pi t}} \exp \left(-\frac{(x-v t)^{2}}{4 D_{x} t}\right) \exp \left(-\frac{y^{2}}{4 D_{y} t}\right) \exp (-\lambda t)
$$

The initial condition and the Dirichlet boundary conditions are defined using the exact solution (47) at starting time $T_{0}=100$ and with $u_{0}=1.0$. We have $\lambda=10^{-5}, v=0.001$ and $D_{x}=0.0001, D_{y}=0.0005$.

In order to develop the computer algorithms for this second example, we work absolutely similarly to the first example. We generalize the adopted scheme for 
one spatial dimension of the first example to a new scheme with two spatial dimensions for the second example. The actual difference is that in this case we decompose both domains of $\Omega, \Omega_{x}=[0,150]$ and $\Omega_{y}=[0,150]$, in two overlapping sub-domains $\Omega_{x, 1}=\left[0, L_{2}\right]$ and $\Omega_{x, 2}=\left[L_{1}, L\right]$, where $L_{1}<L_{2}$, and $\Omega_{x, 1} \bigcap \Omega_{x, 2}=\left[L_{1}, L_{2}\right]$ is the overlapping region for $\Omega_{x, 1}$ and $\Omega_{x, 2}$. We work similarly for $\Omega_{y, 1}$ and $\Omega_{y, 2}$. In order to test the algorithms, we select the same overlap sizes in both spatial dimensions $x$ and $y$, which is the number that appears in the row "overlap" of the following two tables. Again, we demonstrate a comparison between the classical method combining A-B splitting with overlapping Schwarz wave form relaxation (Table 3) and our new proposed combined time-space iterative splitting method (Table 4).

\begin{tabular}{||c||c|c||c|c||c|c||}
\hline \hline time-step & err & err & err & err & err & err \\
\hline$\Delta t=2.5$ & $1.67 e-3$ & $1.13 e-3$ & $1.95 e-4$ & $1.84 e-4$ & $1.13 e-5$ & $1.21 e-5$ \\
\hline$\Delta t=5$ & $2.32 e-3$ & $2.10 e-3$ & $2.76 e-4$ & $2.82 e-4$ & $3.98 e-5$ & $3.84 e-5$ \\
\hline$\Delta t=10$ & $2.45 e-3$ & $2.18 e-3$ & $4.77 e-4$ & $4.86 e-4$ & $5.39 e-4$ & $4.27 e-4$ \\
\hline \hline Overlap & 30 & 70 & 30 & 70 & 30 & 70 \\
\hline space-step & \multicolumn{2}{|c||}{$h=1$} & \multicolumn{2}{|c||}{$h=0.5$} & \multicolumn{2}{|c||}{$h=0.25$} \\
\hline \hline
\end{tabular}

Table 3. Error for the second example using the classical method for two different sizes of overlapping 30 and 70 .

\begin{tabular}{||c||c|c||c|c||c|c||}
\hline \hline time-step & err & err & err & err & err & err \\
\hline$\Delta t=2.5$ & $1.43 e-3$ & $1.02 e-3$ & $1.74 e-4$ & $1.32 e-4$ & $1.01 e-5$ & $8.22 e-6$ \\
\hline$\Delta t=5$ & $2.19 e-3$ & $1.83 e-3$ & $2.54 e-4$ & $2.38 e-4$ & $3.74 e-5$ & $3.52 e-5$ \\
\hline$\Delta t=10$ & $2.31 e-3$ & $2.02 e-3$ & $4.59 e-4$ & $4.62 e-4$ & $5.17 e-4$ & $4.08 e-4$ \\
\hline \hline Overlap & 30 & 70 & 30 & 70 & 30 & 70 \\
\hline space-step & \multicolumn{2}{|c||}{$h=1$} & \multicolumn{2}{|c||}{$h=0.5$} & \multicolumn{2}{|c||}{$h=0.25$} \\
\hline \hline
\end{tabular}

Table 4. Error for the second example using the proposed method for two different sizes of overlapping 30 and 70 .

\section{Conclusions and Discussions}

We present decomposition methods for differential equations based on iterative and non-iterative methods. The classical idea is to decouple time and space and apply separate decomposition methods, which are for space the overlapping Schwarz wave form relaxation method and for time the A-B operator splitting method. The new method combines the time and space and applies for both the iterative operator-splitting method. We prove the convergence and show its 
stability. The verification of the new method is done by comparing with the traditional domain decomposition and lower order time splitting method. The results show more accurate solutions with respect to time and space. We improve the new method by more overlapping. In the future the iterative operator splitting method can be generalized for multi-dimensional problems and also for non-smooth and nonlinear problems in time and space.

\section{References}

1. W. Cheney. Analysis for Applied Mathematics. Graduate Texts in Mahematics., 208, Springer, New York, Berlin, Heidelberg ,2001.

2. K.-J. Engel, R. Nagel, One-Parameter Semigroups for Linear Evolution Equations. Springer, New York, 2000.

3. I. Farago, J. Geiser. Iterative Operator-Splitting methods for Linear Problems. Preprint No. 1043 of the Weierstrass Institute for Applied Analysis and Stochastics, Berlin, Germany, June 2005.

4. J. Geiser. Discretisation Methods with embedded analytical solutions for convection dominated transport in porous media Proceeding of Numerical Analysis and Applications, Third international conference, Rousse, Bulgaria, 2004, Lect.Notes in Mathematics (Springer), vol.3401, 2005.

5. J. Geiser, O. Klein, and P. Philip. Anisotropic thermal conductivity in apparatus insulation: Numerical study of effects on the temperature field during sublimation growth of silicon carbide single crystals. Preprint Weierstra-Institut fr Angewandte Analysis und Stochastik, Berlin, 2005.

6. W. Hundsdorfer and J.G. Verwer. Numerical Solution of Time-dependent Advection-Diffusion-Reaction Equations. Springer Series in Computational Mathematics, 33, Springer Verlag, 2003.

7. K.H. Karlsen and N.H. Risebro. Corrected operator splitting for nonlinear parabolic equations. SIAM J. Numer. Anal., 37(3):980-1003, 2000.

8. K.H. Karlsen, K.A. Lie, J.R. Natvig, H.F. Nordhaug, and H.K. Dahle. Operator splitting methods for systems of convection- diffusion equations: nonlinear error mechanisms and correction strategies. J. Comput. Phys., 173(2):636-663, 2001.

9. G.I Marchuk. Some applicatons of splitting-up methods to the solution of problems in mathematical physics. Aplikace Matematiky, 1 (1968) 103-132.

10. G. Strang. On the construction and comparision of difference schemes. SIAM J. Numer. Anal., 5:506-517, 1968. 